\title{
Pre-Deflection Routing with Blocking Probability Estimation Scheme in Optical Burst Switch Networks
}

\author{
Jaipal Bisht \\ Ph.D Sch, MANIT \\ Bhopal 462051, India
}

\author{
Aditya Goel, Ph.D \\ Prof., MANIT \\ Bhopal 462051, India
}

\begin{abstract}
Optical burst switching (OBS) technology has been proposed as a promising switching solution for the terabit bandwidth utilization in next-generation wavelength division multiplexing (WDM) optical transport network. Control architecture and Contention resolution are the main issues faced by the Optical Burst Switching networks. This paper only taking care of the Contention problem and to overcome this issue we propose Pre-Deflection Routing with blocking probability estimation Scheme for Optical Burst Switch Networks. In this paper Pre-deflection routing approach has been proposed in which routing is carried out in two ways, Shortest Path First (SPF) and Least Hop First (LHF) Routing to forward the clusters and canoes respectively. Hereafter blocking probability estimation Scheme has been proposed where a forward control packet collects the congestion price and contention price along its paths. If the contention is very high at some particular core nodes then it drops that node and thereafter source node uses this information to revise its burst length and offset time.
\end{abstract}

\section{INTRODUCTION}

\subsection{Optical Burst Network (OBS)}

The progression of optical networking technology AllOptical Network (AON) system is considered as a feasible option for next -era switching networks. With AON system there is no need of O-E-O equipments since optical switches and optical cross connect (OXC) directly works on optical domain which results the data carrying signal can directly transmit in optical domain as well and provides high speed switching $[1,2]$. Optical burst switching (OBS) is an optical networking paradigm that combines the best intrinsic worth of both the circuit switching and packet switching technologies, also because of its high bandwidth utilization and easy realization, OBS is treated as viable solution for providing terabit switching in the near future $[3,4]$. In OBS networks, the first step is to make burst and for this several IP packets having same destination are assembled by using proper burst assembly algorithm into a burst at an ingress node and after that burst is transmitted through core nodes and finally reached at the egress node. At the destination node or egress node the reverse process is applied in which the data burst disassembled back into data packets for the further processing. Moreover for each data burst, the source node sends a control packet or burst header packet on a separate channel .This header packet sets an offset time which is the time gap between the two consecutive data bursts and also reserves a dedicated wavelength for the data burst in advance. When a burst is ready for transmission, the source node sends a control packet toward the destination node on a dedicated control channel to reserve resources at intermediate core nodes along the path [5].

In conventional IP networks, several data packets arrive simultaneously at the source node and source node can deal with only one packet at a time so for the rest of packets buffer is required, OBS can eliminate the need for buffering of the data burst.

The data burst follows after an offset time without waiting for an acknowledgement. The physical separation data bursts and their header packets help to provide flexible electronic processing of headers at optical core nodes and end-to-end transparent all-optical paths for transporting data bursts [6]. OBS also has advantages in switching efficiency, low setup time for bursty IP traffic and in optical hardware feasibility.

In OBS the Control Architecture and the Contention Resolution scheme are two significant issues. The control architecture, defines how an edge node will inform the network about an impending burst and the kind of information about the burst that the source gives to the network. JIT (Just in Time), JET (Just Enough Time), and TSL SP (Time-Space Label Switching Protocol) [6,7], are three of the many signaling schemes proposed for OBS networks. In Optical reservation is a one way process in which a burst starts its transmission without waiting for the reservation acknowledgment and if two or more bursts intend to take the same output port at the same time and on the same wavelength then contention will take place that causes blocking of data bursts, therefore proper contention resolution schemes are needed to be implement.

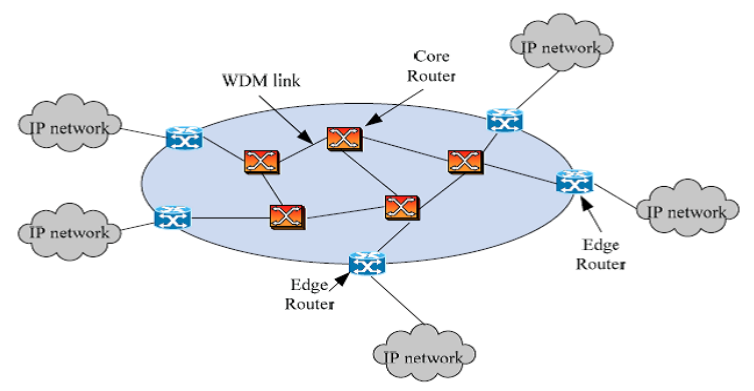

Fig.1 OBS Network Architecture 


\subsection{Issues of OBS Network}

1. An important issue related to one-way reservation in general, and OBS in particular (since there is no optical buffer), is how to deal with contention and reduce burst dropping.

2. Another important issue related to OBS using a non-zero offset time is the end-to-end latency encountered by each burst [3].

3. Certain performance related issues need to be addressed like scheduling, burst aggregation, contention resolution and Quality of services [4].
4. The main design issues include how to interpret the conveyed information and how to react to the current network state.

5. How to provide QoS for users in the optical network [2]

6. Hardware complexity, high cost devices are also various issues.

\subsection{Contention Resolution Schemes in OBS}

The presently used contention resolution schemes can be classified into five domains, but they have some inherent problems as well. Table 1 shows the detail of these schemes.

Table 1: Different contention resolution schemes for OBS network

\begin{tabular}{|c|c|c|c|}
\hline S.N & Domain & Example & Problems \\
\hline 1. & Space & Deflection Routing & $\begin{array}{l}\text { It makes setting the time lag between a burst header } \\
\text { packet and the corresponding data burst i.e. offset time } \\
\text { at the edge node a hard problem because the exact } \\
\text { transmission path of the burst is not known. }\end{array}$ \\
\hline 2. & Wavelength & Wavelength Conversion & $\begin{array}{l}\text { Wavelength converters are expensive and complex } \\
\text { devices. }\end{array}$ \\
\hline 3. & Time & Fiber Delay Line (FDL) & $\begin{array}{l}\text { It will increase data latency and also introduce } \\
\text { complexity for the network. }\end{array}$ \\
\hline 4. & Burst & Segmentation & It is not easy to carry out in the physical layer. \\
\hline 5. & Control & $\begin{array}{l}\text { Shortest-Drop, Look } \\
\text { Ahead Policy }\end{array}$ & $\begin{array}{l}\text { It increases the complexity of implementation too } \\
\text { much. }\end{array}$ \\
\hline
\end{tabular}

\section{PARTIAL PRE - DEFLECTION CONTENTION RESOLUTION SCHEME}

We propose a new scheme to deal with the contention problem in OBS networks by pre-deflecting part of the payload at the edge node. In this scheme traffic payload is divided into two categories, cluster and canoe.

Cluster: It is a consecutive traffic payload consisting of identical attribute data packets.

Canoe: The remainder traffic payload, which is relatively discrete and independent.

This separation of traffic payload is done by Payload Segregator Threshold criterion [8].

\subsection{Payload Segregator Threshold (PST) Criterion}

The function of payload segregator is to categorize the burst assembled by Dual Time Threshold (DTT) assembly algorithm into the cluster and canoe. The criterion of classification is PST, which is an integer value close to the mean value of burst length $l$. If the length of a burst is lower than PST, the burst is classified to the class of canoe; otherwise it belongs to the class of cluster. Let $p$ denote the probability that ST Timer does not interrupt when a new data packet arrives, $x$ denote the inter-arrival time between the new data packet and the anterior one close to it, suppose $\mathrm{x}$ is under independent and identical Pareto distribution, $\mathrm{E}(l)$ is the mean value of burst length $l$. So according to the mechanism of ST Timer, the burst length $l$ obeys geometric distribution $[9,10]$.

So, $\mathrm{E}(l)$ is determined by the Hurst parameter $\mathrm{H}$, when we set the value of $S T$ to be $E$.

$$
\begin{aligned}
& \mathrm{P}=\operatorname{prob}(\mathrm{x}<\mathrm{ST})=\mathrm{F}(\mathrm{ST}) \ldots \ldots \ldots \\
& \text { Prob }(\mathrm{l}=\mathrm{k})=\mathrm{p}^{\mathrm{k}-1}(1-\mathrm{p}) \ldots \ldots(2) \\
& \text { where } \mathrm{k}=1,2 \ldots+\infty \\
& E(l)=\sum_{l=1}^{+\infty} l \cdot p^{l-1} \cdot(1-p)=\frac{1}{1-p}
\end{aligned}
$$

So $\mathrm{E}(l)$ is only determined by the Hurst parameter $\mathrm{H}$ when we set the value of ST to be E.

$$
E(l)=\frac{1}{1-F(E)}=\frac{1}{\left(1-\frac{1}{\alpha}\right)^{2}}=\frac{1}{\{1-1 /(3-2 H)\}^{3-2 H}}
$$

We use the integer value close to E(l) as the value of PST, as shown in Table 1. The load of canoe and load of cluster in the self-similar traffic are:

$$
\text { canoe_load }=\frac{\sum_{l=1}^{P S T} l \cdot p^{l-1} \cdot(1-p)}{E(l)}
$$

And,

$$
\text { cluster_load }=(1-\text { canoe_load })
$$


Table 2. Prob $(x \geq E)$, E(l), PST ,Cluster_Load under Different Degree of Self Similar Traffic

\begin{tabular}{|c|c|c|c|c|c|}
\hline $\boldsymbol{\alpha}$ & $\mathbf{H}$ & $\begin{array}{c}\text { Prob } \\
(\mathbf{x} \geq \mathbf{E})\end{array}$ & $\mathbf{E}(\mathbf{l})$ & PST & Cluster_load \\
\hline $\mathbf{1 . 2}$ & 0.9 & 0.1165 & 8.6 & 9 & 0.6519 \\
\hline $\mathbf{1 . 4}$ & 0.8 & 0.1731 & 5.3 & 6 & 0.6517 \\
\hline $\mathbf{1 . 6}$ & 0.7 & 0.2082 & 4.8 & 5 & 0.6352 \\
\hline $\mathbf{1 . 8}$ & 0.6 & 0.2323 & 4.3 & 5 & 0.6701 \\
\hline $\mathbf{2 . 0}$ & 0.5 & 0.2500 & 4.0 & 4 & 0.6328 \\
\hline
\end{tabular}

By table 2 it is clearly seen that the load of cluster is around $66 \%$, i.e. two-third of the traffic load for the all values of Hurst parameter $(\mathrm{H})$ under Self-Similar traffic.

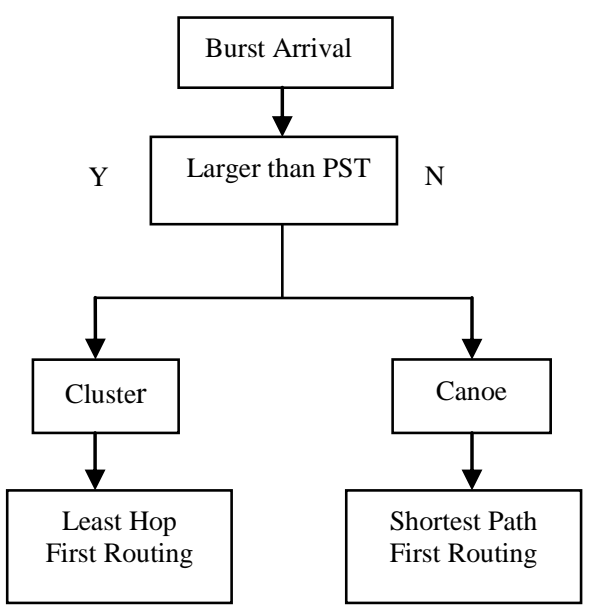

Fig.2 Flow Chart of Payload Segregation

This Payload Segregator Threshold (PST) scheme includes the following two steps:

1. We separate out the traffic payload at the edge nodes into clusters and canoes before they are transmitted through the core nodes.

2. The clusters and canoes will transfer through the core nodes to their destination by the different paths, even though they have the same source address and destination address.

\subsection{Estimation of Blocking Probability}

\section{Technique}

In the OBS networks the all the bursts are transmitted from the source node to destination node through the route of the primary route. The burst reaches all the intermediate nodes until it reaches destination node. If in case all the links are unavailable at any intermediate node or source node, then the burst is deflected onto an alternate route. When the burst is deflected onto an alternate route the preference is given to shorter routes. A burst is considered blocked if it arrives at a given node where all output links are busy or while trying alternate trunks, the burst reaches the maximum allowable number of deflections $[11,12]$.

\subsubsection{Blocking Probability}

Here in this section we describe the estimation of technique to evaluate of blocking probability of OBS networks with deflections between the source node and destination node. So the blocking probability, for bursts with $d \varepsilon(1 \ldots \ldots$ D) deflections, on trunk $\mathrm{j}$ is estimated by [13]:

$\mathrm{BP}_{\mathrm{j}}^{\mathrm{d}}= \begin{cases}L P_{j}\left(C_{j}\right), & \mathrm{d}=0 \\ \sum_{i=T_{j}}^{\mathrm{C}_{j}} L P_{j}\left(l_{i}\right), & \mathrm{d} \geq 1\end{cases}$

Where,

$\mathrm{BP}_{\mathrm{j}}^{\mathrm{d}}$ is the blocking probability with d deflection trunk

Flowchart:

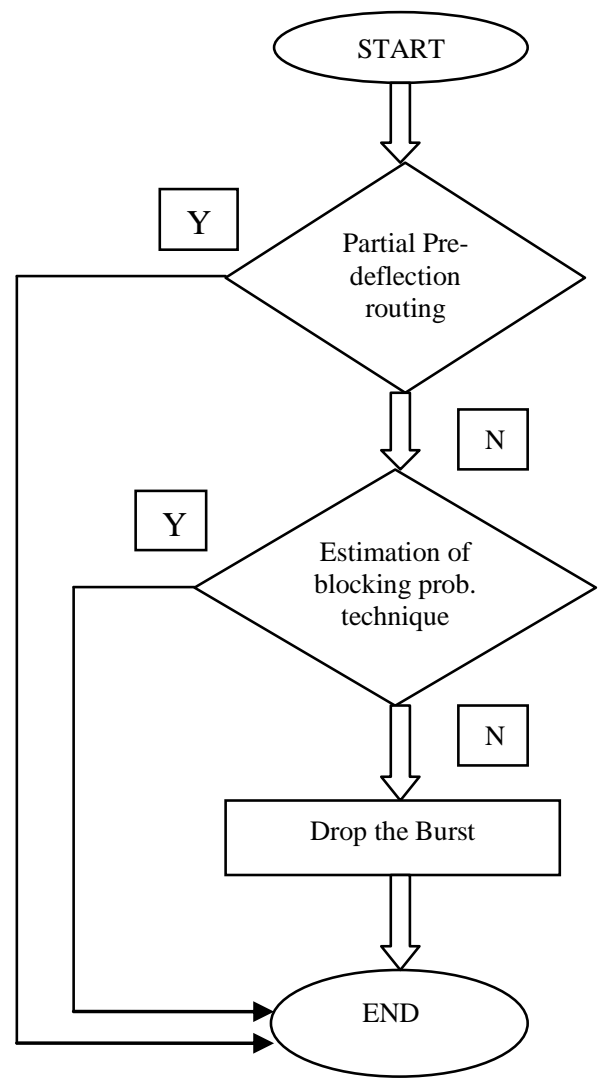

Fig.3 Flow Chart of Our Scheme

\section{SIMULATION RESULTS}

\subsection{Simulation Setup}

In this section, we examine the performance of our Partial Pre-Deflection Routing with Blocking Probability Estimation Scheme for Optical Burst Switch Networks with an extensive simulation study based upon the ns-2 network simulator [14]. We use the OBS network simulator (n-OBS) patch in ns-2, to simulate our network. The simulation settings are given in the following Table 3 . 
Table.3. Simulation settings

\begin{tabular}{|l|l|}
\hline Topology & Mesh \\
\hline Total no. of Nodes & 14 \\
\hline Edge Nodes & 7 \\
\hline Core Nodes & 7 \\
\hline Maximum channels per link & 10 \\
\hline Number of control channels per link & 2 \\
\hline Number of data channels per link & 8 \\
\hline Total channel Bandwidth & $100 \mathrm{Mb}$ \\
\hline Link Delay & $1 \mathrm{~ms}$ \\
\hline Maximum burst size & $40 \mathrm{~Kb}$ \\
\hline Traffic Type & SSIM \\
\hline Packet Size & 512 bytes \\
\hline Low Load & 2 to $10 \mathrm{Mb}$ \\
\hline High Load & 10 to $20 \mathrm{Mb}$ \\
\hline
\end{tabular}

In this simulation, a Constant Burst Rate (CBR) traffic model is used, in which five traffic flows are setup between two edge routers. In all the simulation, the results of our proposed PPDRBE algorithm are compared with the PPDR method.

\subsection{Based on Load}

\section{- $\quad$ Low Load Scenario:}

In low load scenario, we vary the load value from $2 \mathrm{Mb}$ to $10 \mathrm{Mb}$.

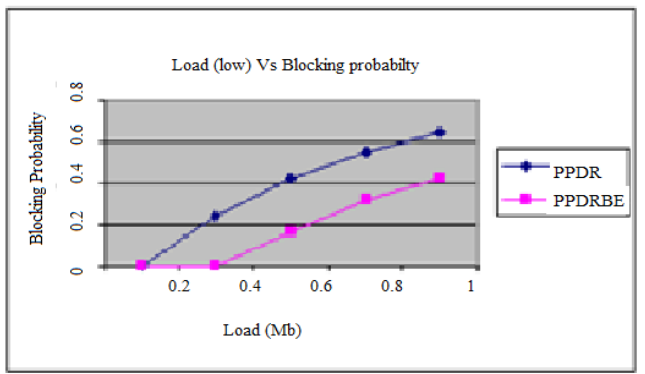

Fig 4: Load (Low) Vs Blocking Probability

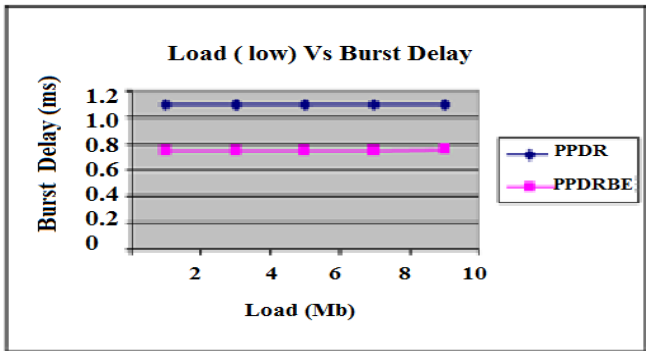

Fig 5: Load (Low) Vs Burst Delay

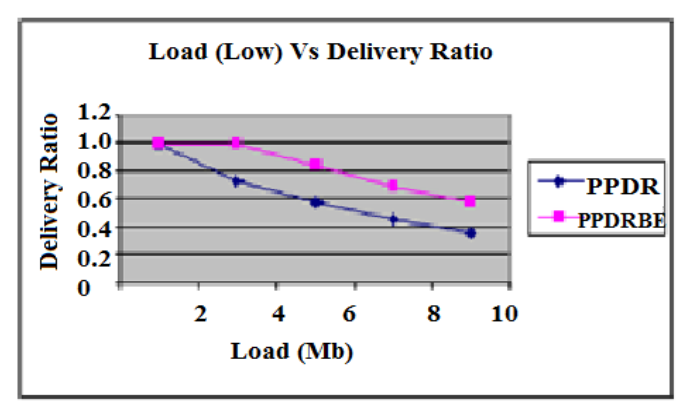

Fig 6: Load (Low) Vs Delivery Ratio

- High Load Scenario:

In high load scenario, we vary the load value from $10 \mathrm{Mb}$ to $20 \mathrm{Mb}$.

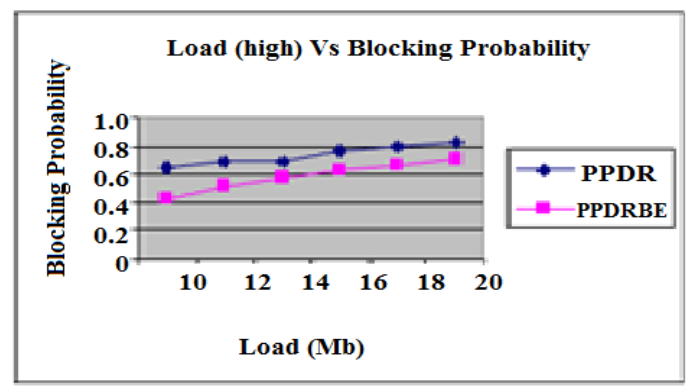

Fig 7: Load (High) Vs Blocking Probability

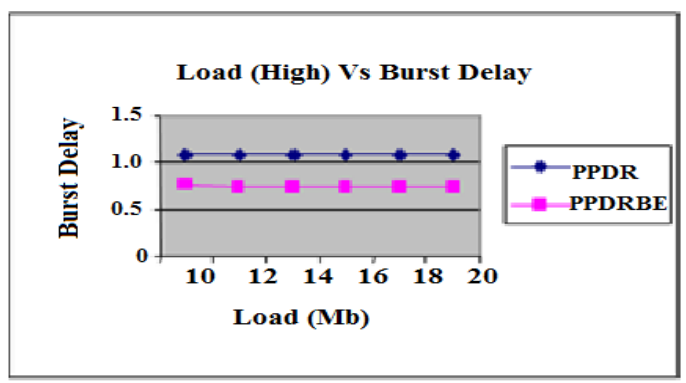

Fig 8: Load (High) Vs Burst Delay

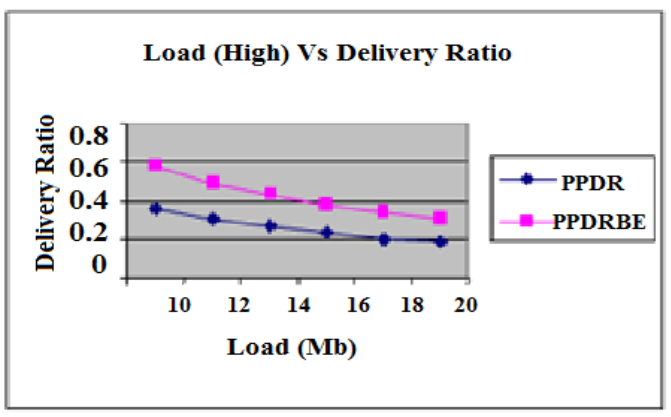

Fig 9: Load (High) Vs Delivery Ratio

From the above results it is clearly shown that in our proposed PPDRBE scheme all the parameters like blocking probability, burst delay and delivery ratio are coming better than the PPDR scheme. 


\section{CONCLUSION}

In this paper we propose Pre-Deflection Routing with blocking probability estimation Scheme for Optical Burst Switch Networks. In this approach Partial Pre-deflection routing approach has been proposed which routing is carried out in two ways Shortest Path First (SPF) routing and Least Hop First Hop First (LHF) routing. The Shortest Path First (SPF) Routing is used by the canoes and LHF routing is used to forward the clusters. Thereafter blocking estimation scheme applied for the calculation of blocking at the different core nodes in which a forward control packet (FCP) collects the congestion price and contention price along its paths, and then this information sent to the source node. The source node uses this information to update its burst length and offset time.

\section{REFERENCES}

[1] C. Qiao and M. Yoo, "Choices, Features and Issues in Optical Burst Switching”, 1999.

[2] Myungsik Yoo, Member, IEEE, Chunming Qiao, Member, IEEE, and Sudhir Dixit, Senior Member, IEEE, "QoS Performance of Optical Burst Switching in IP-Over-WDM Networks", IEEE 2000 .

[3] Jinhui Xu, Chunming Qiao, Jikai Li, and Guang Xu, "Efficient Channel Scheduling Algorithms in Optical Burst Switched Networks", IEEE 2003.

[4] Farid Farahmand, Qiong Zhang and Jason P. Jue, "A Feedback-Based Contention Avoidance Mechanism for Optical Burst Switching Networks", 2004.

[5] Y. Chen, C. Qiao, and X. Yu, "Optical burst switching: A new area in optical networking research," IEEE Network, vol. 18, no. 3, pp. 16-23, May/Jun. 2004.

[6] C. Yuan, Z. B. Li, A. S. Xu, "A Novel Burst Assembly Algorithm for OBS networks--Based On Data-length Time-lag Product" in Asia Pacific Conference on Communication, October 3-5, 2005, Perth, Western Australia. pp. 319-323.
[7] Q. Zhang, V. M. Vokkarane, Y.Wang, and J. P. Jue, "Evaluation of burst retransmission in optical burst switched networks," in Proceedings, IEEE Broadnets 2005, Optical Networking Symposium, October 2005.

[8] Rohit Lamba, Dr.Amit Kumar Garg, "Performance Analysis of Scheduling Algorithms In Optical Burst [9] Switching Networks", International Journal of Advanced Research in Computer Science and Software Engineering 2012.

[9] Liang Shan, Linzhen Xie, Zhengbin Li and Anshi Xu," Partial Pre-Deflection- a novel contention resolution scheme for optical burst switching networks", Optical Engineering 46(7), 075007 (July 2007).

[10] Eric W. M. Wong, Senior Member, IEEE, Jayant Baliga, Moshe Zukerman, Fellow, IEEE, Andrew Zalesky, and Garvesh Raskutti, "A New Method for Blocking Probability Evaluation in OBS/OPS Networks With Deflection Routing", IEEE Journal of Lightwave Technology, Vol. 27, No. 23, December 1, 2009.

[11] Amit Kumar Garg, R S Kaler, "An Efficient Routing Scheme to Reduce Packet Loss in All Optical Networks", Journal of Microwaves, Optoelectronics and Electromagnetic Applications, Vol. 9, No. 2, December 2010.

[12] Won-Seok Park, Minsu Shin, Hyang-Won Lee," A Joint Design of Congestion Control and Burst Contention Resolution for Optical Burst Switching Networks", IEEE Journal of Lightwave Technology, Vol. 27, No. 17, September 1, 2009.

[13] A. Belbekkouche, A. Hafid, M. Tagmouti, and M. Gendreau, "A novel formulation for routing and wavelength assignment problem in OBS networks," in ICC 2010, Cape Town, South Africa, May 2010.

[14] Network Simulator: http:///www.isi.edu/nsnam/ns 